\title{
Frequency Correlation Function of Modal Noise in Multimode Optical Fibers
}

\author{
JUNJI OHTSUBO AND MOTONOBU KOUROGI
}

\begin{abstract}
After transmission of highly coherent light through a multimode optical fiber, we can observe a speckle pattern, namely a modal noise, at its exit face caused by the interference of many different propagation modes. Changes of the source frequency can also cause modal noise variations in time at the exit face. From the statistics of modal noise appearing at the exit face of a fiber, we can study the properties of the fiber and the source. In this paper, we have measured the frequency correlation function of modal noise in multimode optical fiber not only at the exit face of the fiber but also at its nearby point. In the theoretical consideration, the geometrical approximation is used to de rive the frequency correlation function at the detecting position. Some experimental results are also given and compared with the theory.
\end{abstract}

\section{INTRODUCTION}

$\mathrm{W}$ HEN a highly coherent light is launched into a multimode optical fiber, a speckle pattern can be observed at the exit face of the fiber as a result of random interference of many different propagation modes. A speckle pattern appeared at the exit face has a certain statistical nature in space and time. It is caused by the variations of optical phase in the different fiber modes and depends on the turbulence applied to the fiber, such as mechanical stress or temperature variations, and the source frequency changes.

A changing speckle pattern which exits at the output plane of a multimode fiber is called a modal noise. This unwanted noise degrades the error performance of a data link in an optical fiber communication. However, we can derive much of the information about the characteristics of fiber and source from modal noise. The first order statistics of a modal noise for a single source have been discussed by Daino et al. [1], Rawson et al. [2], and Goodman and Rawson [3], while the properties of the second order statistics, especially the temporal and spatial frequency correlation functions of modal noise at the fiber exit face have been investigated by Rawson et al. [4], Freude et al. [5], and Weierholt et al. [6]. Moslehi et al. [7] have estimated the bandwidth of a multimode optical fiber using the frequency correlation function FCF of a modal noise. In the derivation of FCF, a planar waveguide approximation has been used to obtain its analytical form and well explained the experimental results.

Manuscript received May 5, 1988; revised September 28, 1988

The authors are with the Faculty of Engineering, Shizuoka University, Hamamatsu 432, Japan.

IEEE Log Number 8927427
In this paper, the FCF's of modal noise in a multimode optical fiber are investigated at the exit face and its nearby point. Though the multimode optical fiber used in this experiment is a cylindrical step index fiber, we used a planar waveguide approximation to calculate the FCF after the analytical derivation by Rawson et al. [4]. The validity of this approximation is verified by the comparison of the results of the theory with those of the experiment. The theoretical FCF's at the exit face and its nearby points of a fiber are calculated by using a geometrical optics assumption of an effective light source array corresponding to the propagation modes.

We must change the source frequency continuously with time to calculate the FCF in the actual experiment. For such a purpose, we use a semiconductor laser diode operating a single mode as a tunable light source. We can modify the source frequency from several gigahertz to several tens of gigahertz only by a small injection current change to a laser diode. In the actual experiment, the FCF for a step index fiber is obtained by changing an injection current to the laser diode, or equivalently changing the laser oscillation frequency. The experimental results show an excellent agreement with the theoretical ones.

\section{Frequency Correlation Function}

In this section we find the theory of the frequency correlation function FCF at the exit face and its nearby point in multimode optical fibers. The model used in this analysis is the same geometrical approximation of a planar waveguide as the paper of Rawson et al. [4]. The validity of this approximation for a cylindrical step index fiber will be discussed in the Section IV as comparing the theory with the experiment.

Fig. 1 shows the model of a planar dielectric waveguide of width $2 d$, length $L$, and refractive index $n$. A large number of multiply reflected images of the source ranges from $-\theta_{m}$ to $\theta_{m}$ at the fiber exit face (where the angle $\theta_{m}$ is the complement of the fiber's critical angle for the total internal refraction). From a geometrical optics consideration and a mode condition in the fiber, we can assume that each propagating mode originates from an effective source at the entrance face of the fiber having an equal separation $\Delta W=\lambda_{g} L / 4 d, \lambda_{g}=\lambda / n$ being the light wavelength in the guide. Consider a point $Q$ separated a very small distance $z$ from the fiber exit face and also deviated a distance $x$ from the optical axis. 


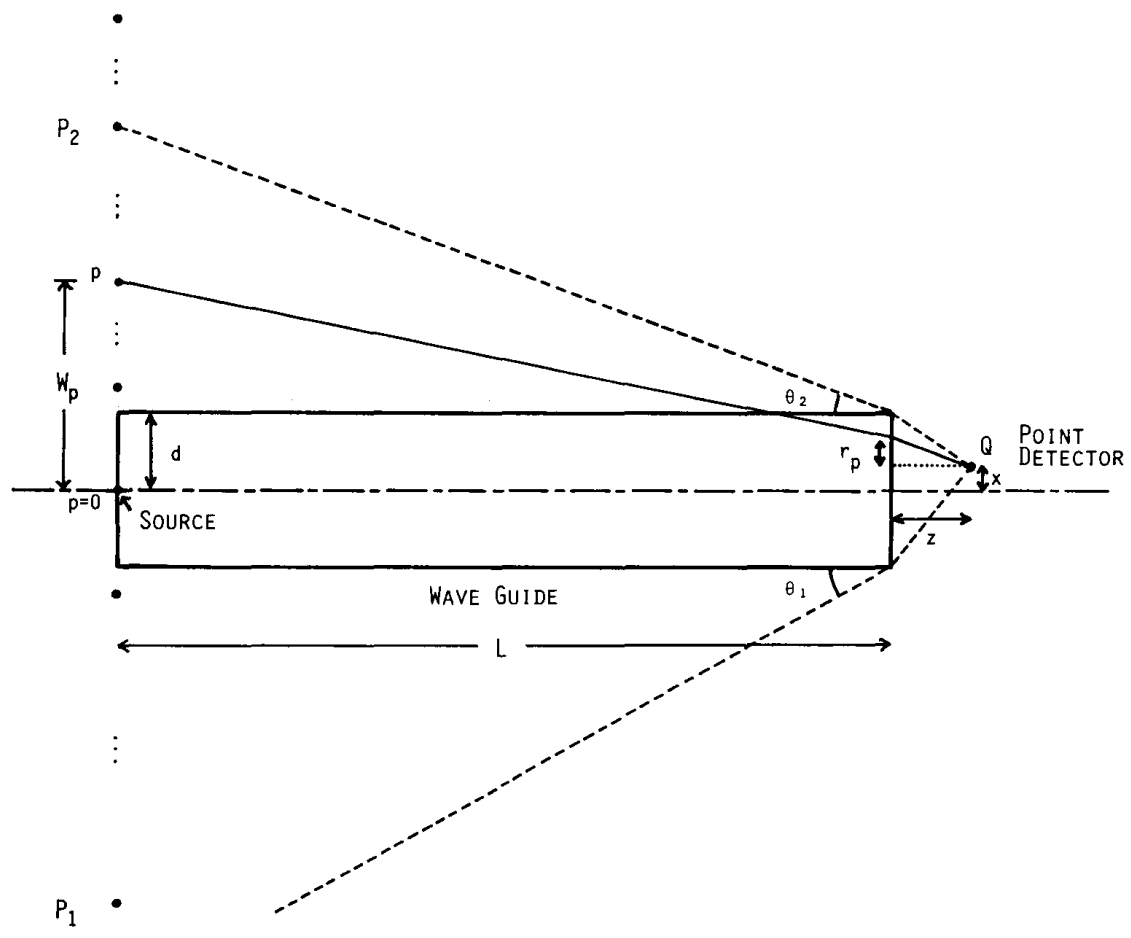

Fig. 1. Model of a planar dielectric waveguide to calculate frequency correlation function.

We will find the FCF at the point $Q$. Since the separation $z$ is very small, we can also use a geometrical approximation to find the light amplitude at the concerning point. With a paraxial approximation, the slant distance from the $p$ th source to $Q$ is

$$
\begin{aligned}
l_{p}= & L+\frac{1}{2 L}\left\{W_{p}-\left(x+r_{p}\right)\right\}^{2}+\Delta l_{p} \simeq L+\frac{W_{p}^{2}}{2 L} \\
& -\frac{W_{p}}{L}\left(x+r_{p}\right)+\Delta l_{p}
\end{aligned}
$$

where

$$
\begin{aligned}
W_{p} & =p \frac{\lambda_{g} L}{4 d} \quad(p: \text { integer }) \\
\Delta l_{p} & =\sqrt{z^{2}+r_{p}^{2}} .
\end{aligned}
$$

The light ray from the $p$ th source is refracted at the point of the exit face separated the distance $x+r_{p}$ from the optical axis and reaches the detecting point $Q$. The field amplitude $U(x, z, \nu)$ at an optical frequency $\nu$ at the point $Q$ is written by a normalized form as follows:

$$
\begin{aligned}
U(x, z, \nu)= & \sum_{p=P_{1}}^{P_{2}} \frac{\beta_{p}}{\sqrt{P_{2}-P_{1}+1}} \\
& \cdot \exp \left[i \frac{2 \pi \nu n}{c}\left(l_{p}+\delta_{p}\right)\right]
\end{aligned}
$$

where $c$ is the velocity of light in vacuum, $\delta_{p}$ represents a random change of the path length suffered by the wave from the $p$ th effective source due to random change of the refractive index caused by flexing or movement of the guide, and $\beta_{p}$ also represents a random change of the amplitude from the $p$ th source. We assume that $\delta_{p}$ and $\beta_{p}$ are independent random variables and they are also independent among each propagation mode.

The field amplitude in (4) is normalized to be unit intensity at the detecting point. $P_{1}$ and $P_{2}$ are the lower and upper numbers of the effective sources to contribute to the field amplitude at the point $Q$ as is shown in Fig. 1. These numbers are defined by

$$
\begin{aligned}
& P_{2}=\frac{d+L \tan \theta_{2}}{\Delta W} \\
& P_{1}=-\frac{d+L \tan \theta_{1}}{\Delta W}
\end{aligned}
$$

where $\theta_{2}$ and $\theta_{1}$ are the maximum refraction angles at the edges in the exit face of the waveguide subtended at the detecting point $Q$ as shown in Fig. 1. When the magnitude of the value $P_{2}$ or $P_{1}$ exceeds the maximum number $P$ of the guided modes, this value should be fixed to the maximum number. $P_{2}$ and $P_{1}$ are always equal or less than the maximum number $P$. This means that the number of the propagating modes to contribute to the amplitude at the observation point is always less than that of the modes at 
the fiber exit face when the detecting point is separated from the plane. The maximum number $P$ is related to the fiber's critical angle $\theta_{m}$ and is written by using the numerical aperture (NA) of the fiber as follows:

$$
P=\frac{\tan \theta_{m}}{\lambda_{g} / 4 d}=\frac{\mathrm{NA} / n}{\lambda_{g} / 4 d} .
$$

To calculate the FCF of the intensity variations, we first find the amplitude correlation function. Substituting (1) into (8) and using the assumption of the independence between the random phase and amplitude, the amplitude frequency correlation function becomes

$$
\begin{aligned}
\Gamma_{U}(\Delta \nu)= & \left\langle U(x, z, \nu) U^{*}(x, z, \nu+\Delta \nu)\right\rangle \\
= & \exp \left(\frac{i 2 \pi \Delta \nu n L}{c}\right) \sum_{p=P_{1}}^{P_{2}} \sum_{q=P_{1}}^{P_{2}} \frac{\left\langle\beta_{p} \beta_{q}^{*}\right\rangle}{P_{2}-P_{1}+1} \\
& \cdot \exp \left[i \frac{\pi c L}{16 n \nu d^{2}}\left(p^{2}-q^{2}\right)\right] \\
& \times \exp \left[i \frac{\pi c L \Delta \nu}{16 n \nu^{2} d^{2}} q^{2}+i \frac{\pi}{2 d}\left\{\left(p r_{p}-q r_{q}\right)\right.\right. \\
& \left.+(p-q) x\}-i \frac{\pi \Delta \nu}{\nu} \frac{x_{q}+r_{q}}{2 d} q\right] \\
& \times \exp \left\{i \frac{2 \pi \nu n}{c}\left(\Delta l_{p}-\Delta l_{q}\right)-i \frac{2 \pi \Delta \nu n}{c} \Delta l_{q}\right\} \\
& \cdot\left\langle\exp \left\{i\left(\phi_{p}-\phi_{q}\right)\right\}\right\rangle \\
= & \exp \left(i \frac{2 \pi \Delta \nu n L}{c}\right) \sum_{p=P_{1}}^{P_{2}} \frac{\left\langle\left|\beta_{p}\right|^{2}\right\rangle}{P_{2}-P_{1}+1} \\
& \cdot \exp \left(i \frac{\pi c L \Delta \nu}{16 n \nu^{2} d^{2}} p^{2}-i \frac{\pi \Delta \nu}{\nu} \frac{x_{q}+r_{q}}{2 d} q\right. \\
& \left.-i \frac{2 \pi \Delta \nu n}{c} \Delta l_{p}\right)
\end{aligned}
$$

where the random phase

$$
\phi_{p}=\frac{2 \pi n \nu}{c} \delta_{p}
$$

is assumed to be uniformly distributed in probability density on the interval $[-\pi, \pi]$ and has a nonzero correlation only at the equal phase $\phi_{p}=\phi_{q}$, i.e., $\left\langle\exp \left\{i\left(\phi_{p}-\right.\right.\right.$ $\left.\left.\left.\phi_{q}\right)\right\}\right\rangle=\delta_{p, q}$. The bracket $\langle\cdots\rangle$ in (8) denotes the operator of the ensemble average. The approximation $1 /(\nu$ $+\Delta \nu) \sim(1-\Delta \nu / \nu) / \nu$ is also used in (8). Since, taking into consideration in the actual experiment, the ratio $\Delta \nu / \nu$ is around $10^{-5},\left(x_{q}+r_{q}\right) / 2 d<1$, and the value of the maximum number $P$ is at most several hundred in step index fiber, the second term in the exponent in the final form of (8) is much less than unity and can be neglected. Furthermore, since the $\Delta l_{p}$ is the order of $100 \mu \mathrm{m}$ and the frequency modulation to a laser oscillation is several tens of gigahertz, the third term is much less than unity as long as the separation $z$ is small, and can also be neglected.
The amplitude $U(x, z, \nu)$ is considered to be a complex Gaussian random process for a sufficiently long waveguide. Therefore, the frequency correlation function of the intensity becomes [8]:

$$
\Gamma_{1}(\Delta \nu)=\left\langle|U(x, z, \nu)|^{2}\right\rangle^{2}\left\{1+\gamma_{1}(\Delta \nu)\right\}
$$

and

$$
\gamma_{1}(\Delta \nu)=\left|\frac{\Gamma_{U}(\Delta \nu)}{\Gamma_{U}(0)}\right|^{2} .
$$

As the random amplitude component $\beta_{p}$ is statistically equal to that of each propagating mode, the FCF of the intensity is written by

$$
\begin{aligned}
& \gamma_{1}(\Delta \nu) \\
& \quad=\left|\frac{1}{P_{2}-P_{1}+1} \sum_{p=P_{1}}^{P_{2}} \exp \left\{i \frac{\pi n L \Delta \nu \tan ^{2} \theta_{m}}{c}\left(\frac{p}{P}\right)^{2}\right)\right|^{2}
\end{aligned}
$$

where the relation of (7) has been used. If there are many modes in the multimode optical fiber, the summation of (12) can be written by an integral form as follows:

$$
\begin{aligned}
\gamma_{1}(\Delta \nu) & =\frac{1}{\left(y_{2}-y_{1}\right)^{2}}\left|\int_{y_{1}}^{y_{2}} \exp \left(i \frac{\pi}{2} u^{2}\right) d u\right|^{2} \\
& =\frac{C^{2}\left(y_{2}, y_{1}\right)+S^{2}\left(y_{2}, y_{1}\right)}{\left(y_{2}-y_{1}\right)^{2}}
\end{aligned}
$$

where

$$
\begin{aligned}
C\left(y_{2}, y_{1}\right) & =\int_{y_{1}}^{y_{2}} \cos \left(\frac{\pi}{2} u^{2}\right) d u \\
S\left(y_{2}, y_{1}\right) & =\int_{y_{1}}^{y_{2}} \sin \left(\frac{\pi}{2} u^{2}\right) d u \\
y_{2} & =\frac{P_{2}}{P} \sqrt{\frac{2 L \Delta \nu(N A)^{2}}{n c}} \\
y_{1} & =\frac{P_{1}}{P} \sqrt{\frac{2 L \Delta \nu(N A)^{2}}{n c}} .
\end{aligned}
$$

When the intensity is detected at the exit face $z=0$ of the fiber, this equation reduces to the obtained result by Rawson et al. [4]. However, their result has lacked the term of the Fresnel sine integral part in (13). We compare the obtained theoretical result with the experiments in the following section.

\section{EXPERIMENTS}

Fig. 2 shows the experimental setup based in the FCF measurements. The light source is a single mode AlGaAs channeled-substrate planar-type laser diode of Hitachi HL7801E with a wavelength $\lambda$ of $0.788 \mu \mathrm{m}$ at a $65-\mathrm{mA}$ injection current and a $25^{\circ} \mathrm{C}$ temperature. The operating condition of the laser oscillation is chosen to minimize the effects of mode hoppings on the FCF measurement by 


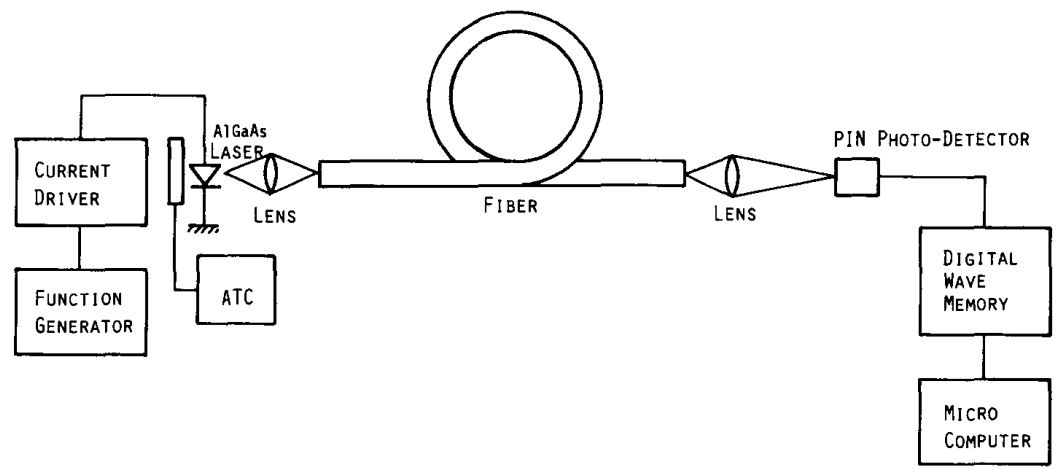

Fig. 2. Experimental setup used to measure the frequency correlation function for step index fiber.

driving an automatic temperature controlled assembly (ATC) attached to a Peltier element. The ATC can regulate the laser diode mount temperature within $\pm 0.04^{\circ} \mathrm{C}$ at an expected value. The frequency of the laser diode can be continuously changed by varying the injection current. To measure the FCF the injection current is modulated by a $10-\mathrm{Hz}$ triangular wave and the modulation amplitude of the injection current is chosen as $10 \mathrm{~mA}$ of peak-to-valley scale at a bias current $65 \mathrm{~mA}$ (the ratio of the bias current to the lasing threshold is $I / I_{\mathrm{th}}=1.5$ ) and a temperature $25^{\circ} \mathrm{C}$. By the triangular modulation, the frequency of the laser oscillation is linearly modulated. There is no mode hopping of the laser frequency between the injection current ranging from 60 to $70 \mathrm{~mA}$ at the temperature $25^{\circ} \mathrm{C}$ in this experiment.

We must find the modulation ratio $\alpha$ of the laser frequency per unit current to estimate the FCF. Since the modulation of the injection current is low frequency in our experiment, the frequency change of the laser oscillation is originated from the temperature modulation effect caused by an injection current change and is also a function of the modulation frequency of the current [9]. The modulation ratio $\alpha$ of the laser frequency is investigated by using an unbalanced Twyman-Green interferometer [10] and is experimentally calculated as $\alpha=2.9$ $\mathrm{GHz} / \mathrm{mA}$ at $10 \mathrm{~Hz}$ modulation frequency of the injection current. Therefore, the total frequency change of the laser oscillation by the full scale current variations becomes 29 $\mathrm{GHz}$ in the experiment.

The fiber to be measured is a step index fiber (Fuji-Opto Co.) of length $4.45 \mathrm{~m}$, core diameter $200 \mu \mathrm{m}$, numerical aperture NA $=0.32$, and refractive index of the core $n$ $=1.50$. The fiber can be considered to have abruptly step index profile which we have assumed in the theoretical treatment. From the value of the numerical aperture of the fiber NA $=0.32$, the total number $2 P$ of the propagation modes at the fiber exit face is calculated as 320 . Since there are many propagation modes in the fiber, the contribution from the effective sources can be assumed to be continuous and the integral approximation used in (13) becomes a good one.

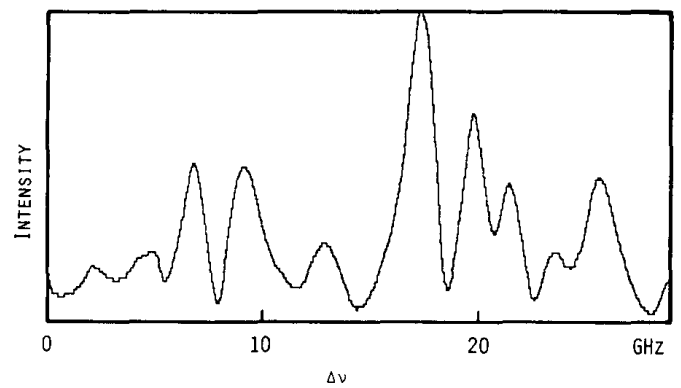

Fig. 3. Example of a modal noise at the exit face of multimode optical fiber produced by the injection current variations.

Fig. 3 shows an example of a speckle pattern, i.e., a modal noise, at the exit face of the multimode optical fiber produced by the injection current variations. A speckle pattern to be measured is magnified by a $\times 100$ microscope objective and detected at its image plane. The speckle intensity variation is detected at the image plane of the fiber exit face by a p-i-n photodiode together with a pinhole much smaller than the size of the image speckle. The detected speckle intensity variation is digitized by 8 bit levels having a 1024 point data length using a digital wave memory. The digitized data in the wave memory is read by a micro computer and is stored in a floppy disk as an original data.

It is noted that there is no mode hoppings as is seen in the figure. The laser power is also modulated by a triangular wave of the injection current. The modulation ratio of the power is about 20 percent under the variations of the injection current ranging from 60 to $70 \mathrm{~mA}$. Because of this modulation, the speckle intensity is not considered as a stationary signal in a strictly statistical sense. However, the speckle signal is assumed to be quasi-stationary if this power modulation can be normalized to have the same mean intensity of every channel of the digitized data.

Two hundred different samples of such a speckle pattern produced by flexing the guide are obtained. After the normalization of speckle intensity in each channel, the 


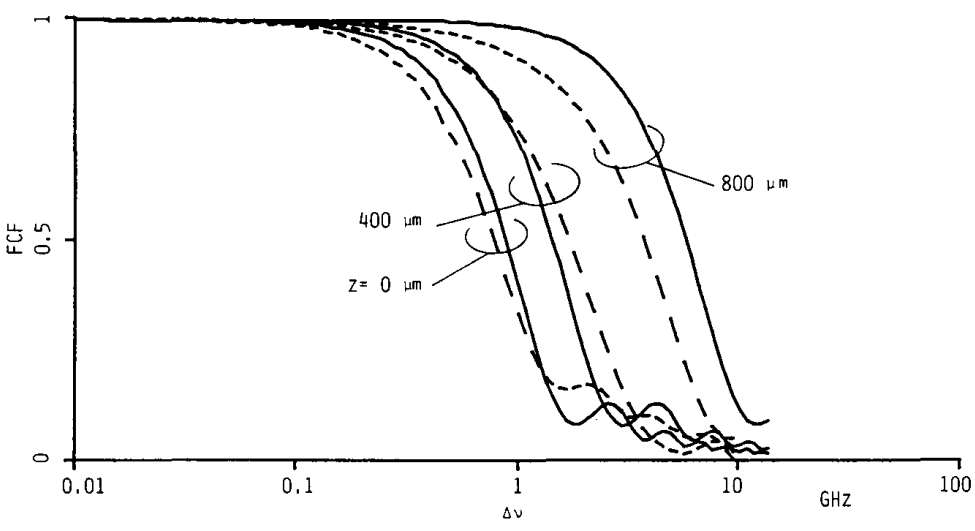

Fig. 4. Frequency correlation function of modal noise for three different detecting points on the optical axis. The solid and broken curves are the theoretical and experimental results, respectively.

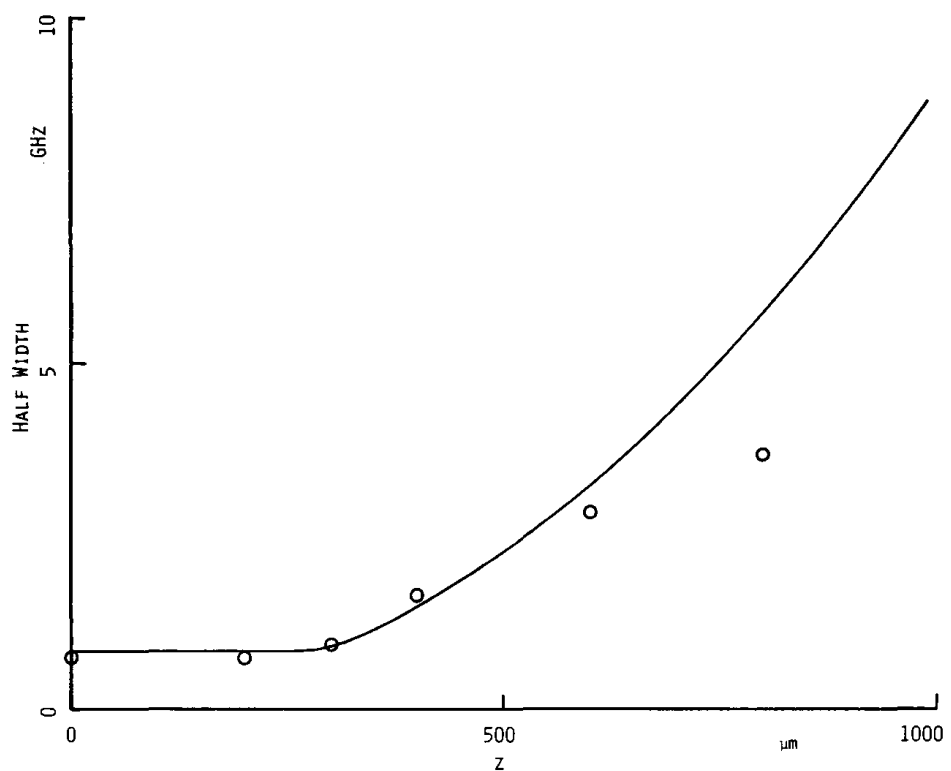

Fig. 5. Width of the half-maximum of the frequency correlation function as a function of the separation $z$. The solid curve is theoretical; the data points are experimental values.

speckle intensity correlation functions are calculated by using a fast Fourier transform algorithm. Finally, each correlation function is averaged out and the averaged FCF of the fiber is obtained. By changing the detecting position of the modal noise separated a certain distance from the fiber exit face, the FCF's are also evaluated as functions of the positions $z$ and $x$.

\section{Results and Discussions}

In this section, we present the results obtained in the measurements of the FCF. Fig. 4 shows the FCF's of a speckle pattern for the three different detecting points $z$ on the optical axis. As the separation $z$ from the fiber exit face increases, the width of the FCF increases. This means the reduction of the number of the effective sources to contribute to the detecting point. As the result of the limitation of the higher propagation modes from the fiber exit face or equivalently the reduction of the effective numerical aperture at the point, the bandwidth of a transmission signal seems to be increased. In this figure, the broken and solid lines represent the experimental results and the theoretical predictions in (13), respectively. The experimental results shows a good agreement with the theoretical ones in spite of the planar waveguide approximation. However, there is a slight disagreement of the curve cor- 


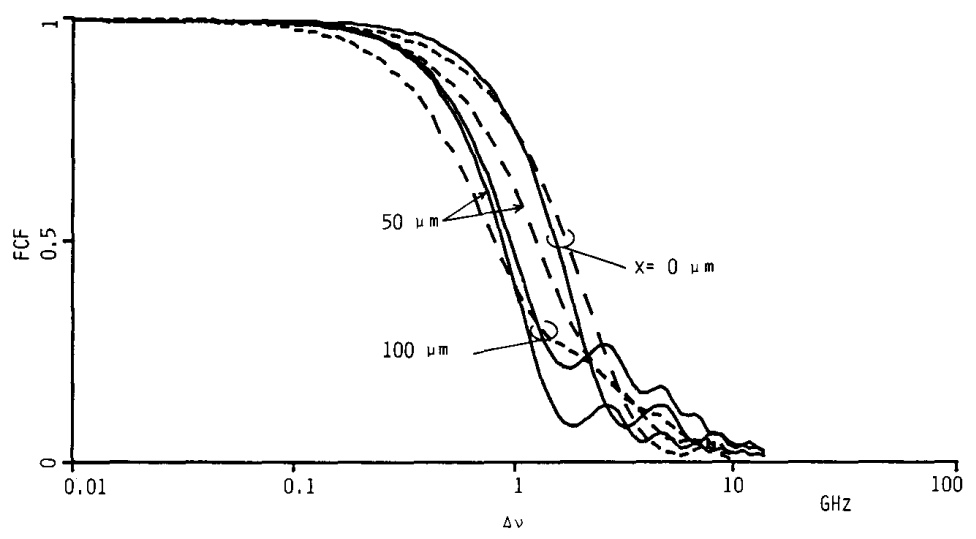

Fig. 6. Frequency correlation function of modal noise for three different detecting points from the optical axis. The separation $z$ from the fiber exit face is fixed as $z=400 \mu \mathrm{m}$. The solid and broken curves are the theoretical and experimental results, respectively.

responding to $z=800 \mu \mathrm{m}$ with the theoretical calculation. Since the approximation used to calculate the FCF is a geometrical one, this disagreement may be on account of the limitation of the approximation.

The width of the half-maximum of the FCF at the exit face is about $1 \mathrm{GHz}$ in this figure. The fiber length is $L$ $=4.45 \mathrm{~m}$ in this experiment. Then the width of the FCF per unit length of the fiber is about $4.45 \mathrm{MHz} \cdot \mathrm{km}$, which is considered to be directly proportional to the $3-\mathrm{dB}$ bandwidth of the fiber transmission.

Fig. 5 shows the width of the half-maximum of the FCF as a function of the separation $z$ from the fiber exit face. The solid line is the theoretical curve of the width of the half-maximum evaluated from (13), while the circles represent the experimental results. The theoretical curve agrees well with the experimental results in the small separation $z$. It is noted here again that the approximation used here becomes worse as the separation $z$ becomes large.

Fig. 6 shows the FCF of the speckle patterns for three different detecting points $x$ from the optical axis, while the separation $z$ from the fiber exit face is fixed as $z=$ $400 \mu \mathrm{m}$. The solid curves represent the theoretical prediction in (13) and the broken curves denote the experimentally obtained FCF at those positions. We can see from this figure that the width of the frequency correlation function becomes maximum on the optical axis. However, it is not clear to find the dependence of the FCF on the coordinate $x$. Hence, to clarify its dependence on the detecting point, the width of the half-maximum of the FCF is plotted against the coordinate $x$.

Fig. 7 shows the dependence of the width of the halfmaximum against the coordinate $x$. The solid curve is again the theoretical prediction in (13) and the circles are the widths of the experimentally obtained FCF. The dotted line in the figure denotes the spatial cutoff (or equiv-

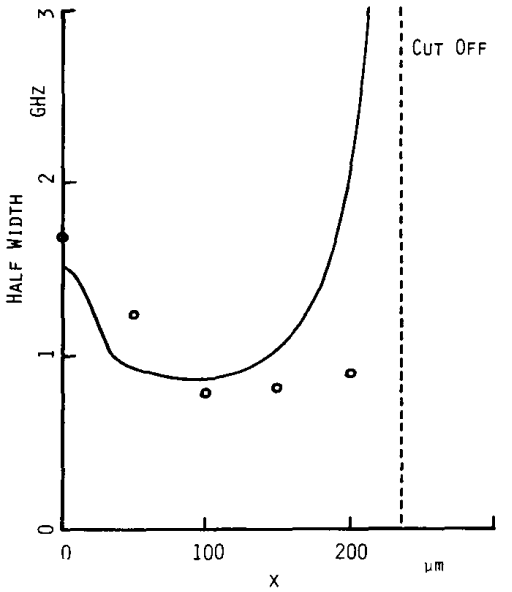

Fig. 7. Dependence of the width of the half-maximum against the coordinate $x$. The solid curve is theoretical; the data points are experimental values

alently effective numerical aperture) at the observation plane $z=400 \mu \mathrm{m}$ predicted by the geometrical optics approximation introduced in Section II. In the experiment, the width of the FCF takes its maximum value on the optical axis. It decreases as the separation $x$ from the optical axis increases and again increases slightly in the region of the larger separation $x$. On the other hand, the theoretical result predicts remarkable increase of the width toward the effective aperture cutoff. The discrepancy between the theory and the experiment in this region may be recognized by the consideration of the diffraction effect. Anyway, the theoretical and experimental results quantitatively coincide with each other except for the detecting position near the spatial cutoff region. It is noted that the number of the propagation modes is small at the 


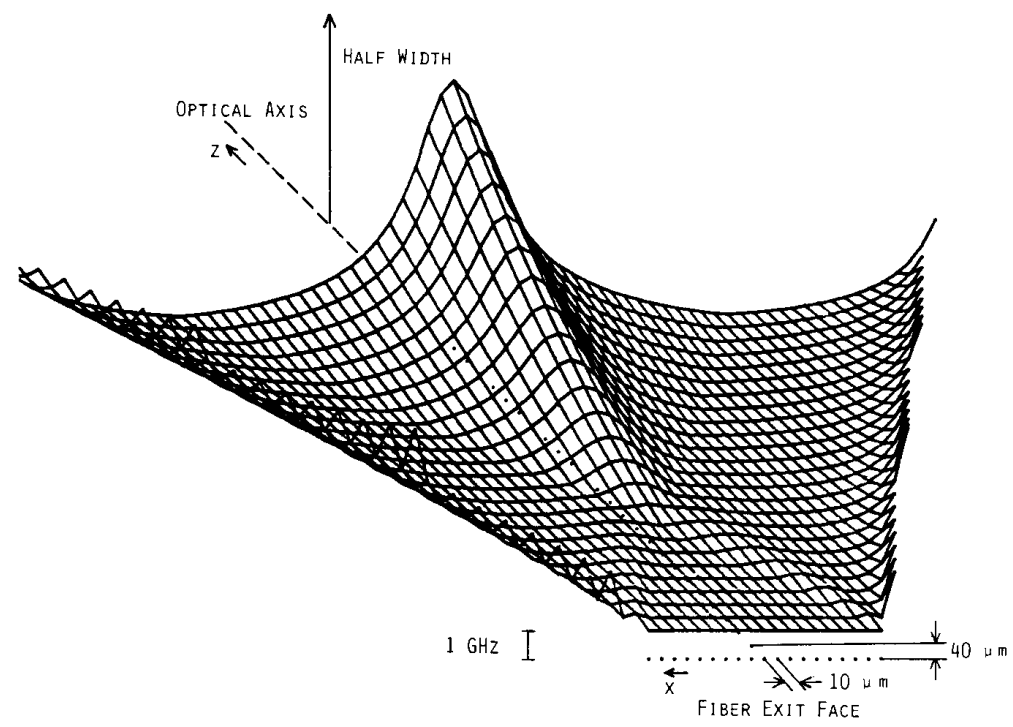

Fig. 8. Dependence of the width of the half-maximum against the coordinates $x$ and $z$.

optical axis and it increases as the deviation $x$ increases. Then, it again becomes small at the edge of the radiation pattern from the fiber exit.

Finally, to see the dependence of the width of the FCF on the detecting position near the fiber exit face, the width of the half-maximum calculated from (13) is shown as a three dimensional plot in Fig. 8. In this figure, the front line denotes the width at the fiber exit face. We can recognize the dependence of a time-varying modal noise due to the source variations on the detecting position.

\section{Conclusions}

We have discussed the frequency correlation function FCF of a modal noise appeared at the exit face and its nearby point of a multimode optical fiber. The experimental results have been compared with the theory of the geometrical approximation based on the model of a planar waveguide. The geometrical approximation can explain the experimental results at certain extent but have a some limitation. It is shown that the approximation is well work when the detecting position is located in a very nearby point of fiber exit face and also the paraxial region. The discrepancies at the far point from a fiber exit face and the edge of the aperture cutoff may be compensated by introducing the diffraction effect.

The dependence of the FCF on the detecting point has also been discussed as a function of fiber parameters. The knowledge of the frequency dependence of modal noise is of special importance as is discussed in [4]. For example, the frequency of a laser source chirps results a modal noise which degrades the error performance of a fiber data link. The changes in error rate can be derived from the prediction of the modal noise statistics. The informations about the fiber parameters and the spectrum of the source frequency can be obtained from the measurement of the FCF. As the FCF is directly proportional to the bandwidth of fiber signal transmission, it may be a good estimate of the bandwidth, especially for a short fiber length.

\section{ACKNOWLEDGMENT}

The authors would like to thank Y. Ishii for valuable discussions and advice.

\section{REFERENCES}

[1] B. Daino, G. DE Marchis, and S. Piazzolla, "Analysis and measurement of modal noise in an optical fibre," Electron. Lett., vol. 15, pp. 755-756, 1979.

[2] E. G. Rawson, J. W. Goodman, and R. E. Norton, "Analysis and measurement of the modal-noise probability distribution for a stepindex optical fiber,"' Opt. Lett., vol. 5, pp. 257-358, 1980.

[3] J. W. Goodman and E. G. Rawson, "Statistics of modal noise in fibers: A case of constrained speckle," Opt. Lett., vol. 6, pp. 324326,1981 .

[4] E. G. Rawson, J. W. Goodman, and R. E. Norton, "Frequency dependence of modal noise in multimode optical fibers," $J$. Opt. Soc. Amer., vol. 70, pp. 968-976, 1980.

[5] W. Freude, C. Fritzsche, G. Grau, and L. Shan-Da, "'Speckle interferometry for spectral analysis of linear sources and multimode optical waveguides," J. Lightwave Technol., vol. LT-4, pp. 64-72, 1986.

[6] A. J. Weierholt, E. G. Rawson, and J. W. Goodman, "Frequency correlation properties of optical waveguide intensity pattern," J. Opt. Soc. Amer. A, vol. 1, pp. 201-205, 1984.

[7] B. Moslehi, J. W. Goodman, and E. G. Rawson, "Bandwidth estimation for multimode optical fibers using the frequency correlation function of speckle patterns, " Appl. Opt., vol. 22, pp. 995-999, 1983.

[8] J. C. Dainty, Ed., Laser Speckle and Related Phenomena, 2nd ed. New York: Springer-Verlag, 1984. 
[9] S. Kobayashi, Y. Yamamoto, M. Ito, and T. Kimura, "Direct frequency modulation in AlGaAs semiconductor lasers," IEEE J. Quantum Electron., vol. QE-18, pp. 582-595, 1982.

[10] Y. Ishii, J. Chen, and K. Murata, "Digital phase-measuring interferometry with a tunable laser diode," Opt. Lett., vol. 12, pp. 233-235. 1987.

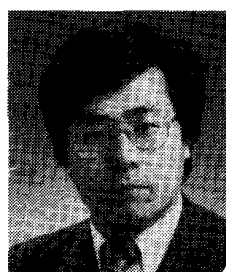

Junji Ohtsubo was born in Oita, Japan. He received the B.S. degree in electronics from Kyusyu Institute of Technology in 1973 . He received the M.S. and Ph.D. degrees in electronics from Hokkaido University in 1975 and 1978 , respectively. In 1978, he joined the Mechanical Engineering Laboratory. During 1981-1982, he was a Research Associate at the Institute of Optics, University of Rochester. Since 1985, he has been an Associate Professor at Shizuoka University. His current research interests are statistical optics, speckle, LDV, active interferometry, and optical information processings.
Dr. Ohtsubo is a member of the Optical Society of America, PhotoOptical Instrumentation Engineers, the Japanese Society of Applied Physics, and the Laser Society of Japan.

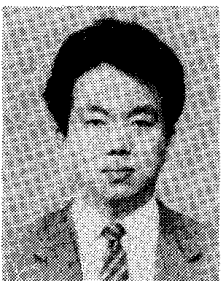

Motonobu Kourogi was born in Shizuoka, Japan. He graduated from Shizuoka University of Engineering in 1988. He is now a graduate student of Tokyo Institute of Technology, where he is engaged in research on noise reduction of a semiconductor laser.

Mr. Kourogi is a member of the Japanese Society of Applied Physics. 